\title{
The ESRC Strategic Network for Obesity: tackling obesity with big data
}

\author{
Michelle A. Morris $\mathbb{D}^{1} \cdot$ Mark Birkin $^{2}$
}

Received: 27 April 2018 / Revised: 24 July 2018 / Accepted: 30 July 2018 / Published online: 5 September 2018

(c) Springer Nature Limited 2018

The ESRC Strategic Network for Obesity (here after referred to as the Network) was established in November 2015, with funding from the Economic and Social Research Council in the UK. The primary aim of the Network is to exploit new and emerging forms of data to catalyse an approach to obesity research using big data, which is transdisciplinary, cross-institutional, multi-sector and international.

Obesity has been characterised as a wicked problem [1], which intersects multiple domains including lifestyle and behaviour, local environments, economic circumstances, socio-demographic characteristics and family circumstances. In the past, it has been difficult or impossible for researchers to access suitable intelligence about many of these drivers. The Network has been explicitly focused on identification of the measures necessary to harness new datasets from multiple sources, for example, consumer behaviour, lifestyle and activity, and health outcomes. Initial steps have been taken to frame these sources with data science methods to embrace the notion of complex systems approaches in obesityproducing new proposals to generate evidence for research and policy evaluation. The embedded value within these data and the co-production with public and third sector participants makes this an exceptional opportunity to ensure output is of outstanding policy relevance.

Over the past 3 years, a series of events have been hosted in diverse locations, each combining open research seminars with developmental activities in a workshop format, which have synthesised the interests of individual Network members. The Network engages a variety of academics, policy makers, and industry partners, and fosters an

Michelle A. Morris

m.morris@leeds.ac.uk

1 Leeds Institute for Data Analytics \& School of Medicine, University of Leeds, Leeds LS2 9JT, UK

2 Leeds Institute for Data Analytics \& School of Geography, University of Leeds, Leeds LS2 9JT, UK inclusive environment with capacity building at its heart by explicit sharing of organisational tasks and contributions from a range of career stages. This is critically important in an area developing as quickly as the field of big data and obesity research where experience of the application area is crucial in equal measures with a fresh approach and new methods development.

\section{Network investigators and members}

\section{Network investigators}

- Professor Mark Birkin (PI), Professor of Spatial Analysis and Policy and Director of the Consumer Data Research Centre (CDRC) and Leeds Institute for Data Analytics (LIDA), University of Leeds

- Dr. Michelle Morris (Director), University Academic Fellow, Leeds Institute for Data Analytics and Leeds Institute for Biomedical and Clinical Sciences, University of Leeds

- Dr. Claire Griffiths, Reader, School of Sport, Leeds Beckett University

- Professor Steven Cummins, Professor of Population Health \& NIHR Senior Fellow, Department for Social and Environmental Health Research, London School of Hygiene and Tropical Medicine

- Dr. Pablo Monsivais, Programme lead-Dietary Public Health Research, Centre for Diet and Activity Research, University of Cambridge

- Professor Jamie Pearce, Professor of Health Geography, Centre for Research on Environment Society and Health, School of Geosciences, University of Edinburgh

\section{And network members}

- Dr. Seraphim Alvanides, Urban Social Geographer, University of Northumbria 
- Dr. Maria Bryant, Senior Research Fellow \& NIHR Career Development Fellow, Leeds Institute of Clinical Trials Research, University of Leeds

- Dr. Thomas Burgoine, Career Development Fellow at the Centre for Diet and Activity Research, University of Cambridge

- Professor Janet Cade, Professor in Nutritional Epidemiology and Public Health, University of Leeds

- Mr. Michael Chang, Project and Policy Manager at the Town and Country Planning Association

- Professor Graham Clarke, Professor of Business Geography at the University of Leeds

- Dr. Darren Dahly, Senior Post-doctoral Researcher at University College Cork, Ireland

- Professor Chris Dibben, Director of the Administrative Data Research Centre for Scotland and the Longitudinal Studies Centre Scotland, Chair in Geography at the University of Edinburgh

- Professor Adam Drewnowski, Professor of Epidemiology and the Director of the Center for Public Health Nutrition at the School of Public Health, University of Washington, Adjunct Professor of Medicine and Joint Member of the Fred Hutchinson Cancer Research Center in Seattle

- Dr. Kimberley Edwards, Course Director, Sports and Exercise Medicine, Faculty of Medicine \& Health Sciences

- Dr. Ellen Flint, Lecturer and MRC Population Health Strategic Skills Fellow at the London School of Hygiene and Tropical Medicine

- Ms. Anna Frearson, Consultant in Public Health at Leeds City Council, Leeds

- Professor Paul Gately, Director of MoreLife and a Professor of Exercise and Obesity at Leeds Beckett University and Visiting Professor in the Department of Surgery and Cancer at Imperial College London

- Dr. Mark Green, Lecturer in Health Geography, University of Liverpool

- Dr. Darren Greenwood, Senior Lecturer in Biostatistics, University of Leeds

- Dr. Emily Henderson, Medical Anthropologist, Lecturer and Senior Research Associate in the Centre for Public Policy and Health in the School of Medicine, Pharmacy and Health, Durham University

- Dr. Amelia Lake, Dietitian and Public Health Nutritionist, Lecturer in Knowledge Exchange in Public Health at Durham University and fuse, the UKCRC Centre for Translational Research

- Dr. Daniel Lewis, Research Fellow in the Department of Social and Environmental Health Research at London School of Hygiene and Tropical Medicine

- Dr. Robin Lovelace, University Academic Fellow, Institute for Transport Studies, University of Leeds
- Dr. Nick Malleson, Associate Professor in Geographical Information Systems, University of Leeds

- Mr. Paul Niblett, Section Head in the lifestyles team within the Health and Social Care Information Centre (HSCIC-now NHS Digital)

- Dr. Duncan Radley, Senior Research Fellow in Physical Activity and Obesity at Leeds Beckett University

- Professor Pinki Sahota, Professor in Nutrition and Childhood Obesity within the Institute for Health and Wellbeing at Leeds Beckett University and Chair of the Association for the Study of Obesity (ASO)

- Professor Jaap Seidell, Professor of Nutrition and Health, and Director of the Institute for Health Sciences at the School of Health \& Life Sciences at the Vrije Universiteit Amsterdam, the Netherlands

- Dr. Niamh Shortt, Senior Lecturer in Health geography and co-founder of the Centre for Environment, Society and Health (CRESH), School of Geosciences, University of Edinburgh

- Dr. Ian Shuttleworth, Lecturer in Human Geography, Queens University, Belfast

- Professor Alex Singleton, Professor of Geographic Information Science at the University of Liverpool

- Ms. Cheryl Squire, Obesity lead, Public Health Team at Leeds City Council

- Ms. Emma Strachan, Nutrition lead, Public Health Team at Leeds City Council

- Dr. Kate Timmins, Senior Lecturer in Physical Activity and Health, University of Lincoln

- Mr. Andy Thompson, UK Chairman, Anytime Fitness

- Dr. Sandy Tubeuf, Associate Professor in Health Economics, Academic Unit for Health Economics, University of Leeds

- Dr. Christina Vogel, Senior Research Fellow, MRC Lifecourse Epidemiology Unit, University of Southampton

- Ms. Emma Wilkins, PhD Candidate, Leeds Beckett University and University of Leeds

- Dr. James Woodcock, Programme lead - Public Health Modelling, Centre for Diet and Activity Research, University of Cambridge

- Professor Jeremy Wyatt, Professor of Digital Healthcare and Director, Wessex Institute of Health and Research, Faculty of Medicine, University of Southampton

\section{Network events and generation of the paper series}

The programme of events was designed to move progressively through the research lifecycle, from data identification and evaluation through methods and model development to exploitation and policy analysis. 
In the first event (24 November 2015, University of Leeds), the group established a vision embracing policy ambitions and research goals, combined with a realistic appreciation of existing research, and available data initiating dialogue around data requirements and scope of possibilities.

Our second event (16 March 2016, University of Cambridge) capitalised on the unique opportunity to have existing and emerging experts together regularly, establishing a definition of big data in the obesity context, inspired by the Foresight obesity systems map in which to report findings. Methods and models for obesity research from different disciplines were showcased and debated.

The third event (18 May 2016, London School of Hygiene and Tropical Medicine) considered international examples of new research that use data to visualise obesity and related findings, allowing the group to compare and contrast evidence and data relating to different populations in the developed world.

Policy and applications was the focus of the fourth event (13 September 2016, University of Edinburgh). As a multisector network, embracing the experience of policy makers with data-driven research and its influence on practice, to date and in the future is pivotal. Co-production of policy relevant data-driven solutions for real-world scenarios was the focus of this meeting.

The latest event (25 April 2017, Leeds Beckett University) crystallised opportunities and challenges as recurrent themes throughout the previous events. This meeting focused entirely on these, discussing how challenges were overcome and how lessons learned can be shared with the wider community. The need to grasp opportunities was paramount for a range of case studies demonstrated.

In parallel to the series of events, the Network has conducted a proof of concept project considering each of these stages with real population data.

This paper series begins with a review of the literature where obesity research has used big data and a review of the data landscape and its coverage in the context of the Foresight obesity systems map. This will be followed with international examples of big data in obesity research practice along with the development of data-driven tools for policy makers from both the academic and policy maker perspective. Empirical research generated by the Network will culminate the series, accompanied by a report on the issues and obstacles encountered by the Network and how we see the future in this area, as reported by the investigator team.

The network members comprise a multi-disciplinary group with diverse interests in public health, epidemiology, geography, health economics and nutrition. The events have attracted delegates with more fine-grained interests in metabolism and molecular medicine, but the major focus has been on social and environmental circumstances, which are modifiable through public policy intervention. The feasibility of decoupling across scales between the microscopic and the environmental influences will also be debated in the future papers, which concludes this series.

We believe that the work of the network is timely and highlights work done to date, new findings and the potential for the future and are delighted to share this with the International Journal of Obesity and its readers.

Acknowledgements The ESRC Strategic Network for Obesity was funded via Economic and Social Research Council grant number ES/ N00941X/1. The authors would like to thank all of the network investigators (www.cdrc.ac.uk/research/obesity/investigators/) and members (www.cdrc.ac.uk/research/obesity/network-members/) for their participation in network meetings and discussion, which contributed to the development of this paper.

\section{Compliance with ethical standards}

Conflict of interest M.A.M. is inventor and shareholder at Dietary Assessment Ltd. The remaining authors declare that they have no conflict of interest.

\section{References}

1. Groves T. Is obesity a wicked problem?. BMJ. 2008;337:a2548. 